\title{
Estudio de la degradación de elementos refractarios de alúmina en la sinterización de aceros modificados con $\mathrm{Mn}$
}

\author{
J. SICRE-ARTALEJO', M. CAMPOS', J.M. TORRALBA', J. ZBIRAL ${ }^{2}$, H. DANNINGER², P. PENA ${ }^{3}$ \\ 'Departamento de Ciencia e Ingeniería de Materiales e Ingeniería Química, \\ Universidad Carlos III. Avda. de la Universidad 30, 28911, Leganés, Madrid. \\ ${ }^{2}$ Technische Universität Wien, Institut für Chemische Technologien und Analytik, A-1060 Vienna, Austria \\ ${ }^{3}$ Instituto de Cerámica y Vidrio, CSIC. Campus de Cantoblanco, Madrid, España.
}

\begin{abstract}
En este trabajo se estudia la degradación de los refractarios de un horno de sinterización, cuando están involucrados en el proceso aceros de baja aleación con adición de manganeso. Dada la elevada $P_{\text {vapor }}$ del manganeso y las atmósferas habituales de sinterización para este tipo de aceros, se produce su sublimación durante el ciclo térmico. Se proporciona así, una fase gaseosa, que pudiendo ser beneficiosa para la difusión en las muestras de acero, puede alterar la composición de los refractarios. Se sabe que en la corrosión de materiales cerámicos refractarios son muchos los procesos individuales que contribuyen, pero siempre basados en las propiedades físico-químicas del agente corrosivo así como de las propiedades intrínsecas del refractario, como la porosidad interconectada y la presencia de múltiples fases (1). Independientemente del mecanismo de actuación, puede generar productos que alteren el comportamiento del refractario, acelerando su envejecimiento y disminuyendo su vida en servicio. Por ello, se estudia la evolución de los refractarios con el tiempo de exposición a atmósferas que contengan vapor de manganeso a la temperatura usual de sinterización, $1120^{\circ} \mathrm{C}$. Los cambios microestructurales en los refractarios, como consecuencia de la presencia de $\mathrm{Mn}_{(\mathrm{o})^{\prime}}$ se analizan mediante microscopía óptica, electrónica con microanálisis de RX (EDS), difracción de RX y fluorescencia de RX.
\end{abstract}

Palabras clave: Corrosión, Refractarios, Alúmina, Acero, Manganeso.

Study of the degradation of the alumina refractory during the sintering process of steels modified with Mn.

The present work studies the degradation of the refractory bricks for sintering furnace, when steels with Mn are sintered. Due to the high $\mathrm{P}_{\text {vapour }}$ of $\mathrm{Mn}$ and the standard sintering atmospheres for this type of steels, Mn sublimates during the thermal cycle. This sublimation results in a gaseous phase beneficial to the diffusion processes related to the sintering of the steel but it could also alter the refractory's composition. It is known that in the corrosion of refractory ceramics are many the individual processes involved, but always based on the physical-chemical properties of the corrosive agent, as well as the intrinsic properties of the refractory such as the interconnected porosity and the presence of multiple phases (1). Independently of the operating mechanism, new compounds can be produced altering the refractory behaviour, accelerating the ageing and diminishing its working conditions. For this matter, it is studied the evolution of the refractory tiles with the exposure time to Mn containing atmospheres at a standard sintering temperature, $1120^{\circ} \mathrm{C}$. Microstructural changes in the refractory elements, as a consequence of the presence of $\mathrm{Mn}_{(\mathrm{g})}$ are analysed through optical microscopy, scanning electron microscopy with $\mathrm{XR}$ microanalyses (EDS), XR diffraction and XRF fluorescence studies.

Keywords: Corrosion, Refractory, Alumina, Steel, Manganese.

\section{INTRODUCCIÓN}

Las altas prestaciones que actualmente demanda el sector del automóvil están haciendo que se intensifiquen los esfuerzos en mejorar el comportamiento en servicio y reducir los costes de los aceros sinterizados. Las propiedades finales de los componentes sinterizados y su coste dependen de dos factores principales, la densidad y el sistema de aleación. El renovado interés del Mn como elemento de aleación es debido tanto al precio, como al nivel excelente de propiedades que se pueden conseguir tanto en estado sinterizado como después de los tratamientos térmicos, independientemente de su elevada afinidad por el oxígeno que lo había relegado de la pulvimetalurgia (2-5).

Son muchas las ventajas metalúrgicas que ofrece la incorporación de este elemento de aleación, además de su precio, como recoge uno los primeros trabajos publicados sobre este elemento de aleación (6). Dada la alta presión de vapor de este elemento, a las temperaturas habituales de sinterización, sublima dando lugar al así llamado "efecto de auto-limpieza del $\mathrm{Mn}^{\prime \prime}(7,8)$. Los primeros estudios relacionados con los procesos de evaporación y condensación del manganeso durante la sinterización, indican que la sublimación y la condensación de los vapores de manganeso tienen lugar de manera efectiva a partir de $\operatorname{los} 700^{\circ} \mathrm{C}$, tanto en atmósferas mixtas de $\mathrm{N}_{2}-\mathrm{H}_{2}$ como en $\mathrm{H}_{2}$ puro, y son fenómenos estrechamente relacionados con el tamaño de partícula de este $(9,10)$. Se modifican así, los agentes reductores presentes en la atmósfera de sinterización y se postulan dos posibles comportamientos según describen las reacciones descritas en las ecuaciones [1], [2] y [3]. 
Salak propone en (9) que parte del vapor de Mn puede oxidarse con el oxígeno de la atmósfera, retirando oxígeno de ella y reduciendo su presión parcial, contribuyendo a reducir el punto de rocío y facilitando la sinterización de los aceros de baja aleación. Si se considerase el diagrama de Ellingham-Richardson, para conseguir la reducción del Mn-O se necesitarían puntos de rocío cercanos a los $-55^{\circ} \mathrm{C}$ a $1120^{\circ} \mathrm{C}$. Sin embargo, gracias a las reacciones descritas en [4], [5] y [7], la sinterización se realiza de manera efectiva con puntos de rocío de $-30^{\circ} \mathrm{C}$.

$2 \mathrm{Mn}(\mathrm{g})+\mathrm{O}_{2} \leftrightarrow 2 \mathrm{MnO}$

Reacción principal propuesta por A. Salak (7)

$\mathrm{Mn}(\mathrm{g})+\mathrm{H}_{2} \mathrm{O} \leftrightarrow \mathrm{MnO}+\mathrm{H}_{2}$

Reacción principal propuesta por A. Cias (11)

$\mathrm{MO}+\mathrm{H}_{2} \leftrightarrow \mathrm{M}+\mathrm{H}_{2} \mathrm{O}$

$\mathrm{MO}+\mathrm{C} \leftrightarrow \mathrm{M}+\mathrm{CO}$

$\mathrm{C}+\mathrm{CO}_{2} \leftrightarrow 2 \mathrm{CO}$

$\mathrm{C}+\mathrm{H}_{2} \mathrm{O} \leftrightarrow \mathrm{H}_{2}+\mathrm{CO}$

$\mathrm{MO}+\mathrm{CO} \leftrightarrow \mathrm{M}+\mathrm{CO}_{2}$

Según propone (11), a temperaturas superiores a $920^{\circ} \mathrm{C}$, el Mn sublimado puede reaccionar con el vapor de agua presente en la atmósfera de sinterización, oxidarse y producir $\mathrm{H}_{2}$, según la reacción mostrada en [2], aumentando el carácter reductor de la atmósfera, según describe [3].

$\mathrm{Al}$ incorporar el carbono en los aceros en forma de grafito, se suministra otro agente reductor, que interacciona tanto con el polvo metálico [7] como con la atmósfera de sinterización [4-6]. Por todo ello, es posible realizar la sinterización en atmósferas con puntos de rocío estándar, sin necesidad de una elevada pureza, favoreciendo de este modo la continuidad del proceso y por tanto su industrialización. Por lo tanto, es de especial interés el estudio del efecto que pueda tener la presencia de $\mathrm{Mn}_{(\mathrm{g})}$ que se forma durante la sinterización sobre los refractarios del horno.

Puesto que los procesos en los que se usan los materiales cerámicos refractarios se alcanzan temperaturas muy elevadas, la corrosión asociada a aquellos es de gran complejidad. Dicho ataque es el resultado de numerosos procesos individuales, todos ellos basados por una parte en las propiedades intrínsecas del propio material refractario, y también en las propiedades físico-químicas del agente externo.

A la hora de estudiar procesos en los que estén involucrados materiales cerámicos refractarios, se tiene que considerar que éstos tienen una porosidad interconectada importante y que además, en su mayoría son polifásicos lo que favorece un ataque preferencial. El hecho de poseer una alta porosidad interconectada facilita el acceso del agente externo y aumenta la cantidad de superficie reactiva, favoreciendo la corrosión.

Una herramienta muy útil en el estudio de procesos de corrosión, de una complejidad moderada, en refractarios son los diagramas de fases puesto que permiten determinar los mecanismos de ataque que predominan durante el proceso y sus consecuencias. No obstante, existen otros fenómenos relevantes que se producen paralelamente al ataque que no aparecen en estos diagramas. Por ejemplo, los cambios de volumen asociados a las transiciones de fase, que pueden hacer aumentar o disminuir, la superficie expuesta al ataque del agente externo.

Dependiendo de por dónde se produzca el ataque del agente externo, se han definido en $(12,13)$ tres mecanismos de corrosión diferentes: disolución superficial, penetración en borde de grano y/o a través de la porosidad interconectada. Dichos mecanismos se pueden dar bien de una manera aislada o combinada. En este trabajo se estudia el tipo de ataque que produce el agente externo $(\mathrm{Mn}(\mathrm{g}))$ y los productos de reacción sobre un refractario de alúmina esferoidal. El corindón es uno de los compuestos más estables en atmósfera reductora, sólo es más estable el CaO. Por otro lado, para que reaccione con el $\mathrm{N}_{2}$ y forme nitruro de aluminio $\mathrm{u}$ oxinitruro de aluminio es necesaria la presencia de carbón activo por lo que a la temperatura de sinterización en la atmósfera del $\mathrm{N}_{2}$ / $\mathrm{H}_{2}$ que se ha utilizado para hacer los ensayos de corrosión no es de esperar ninguna reacción entre el $\mathrm{N}_{2} \mathrm{y} / \mathrm{o}$ el $\mathrm{H}_{2}$ con el refractario (14).

Además, tal y como se describe en (13), la corrosión que sufre un material cerámico debido a la exposición a un agente corrosivo externo, se traduce en una degradación física y química de aquél y además, dicho proceso, depende de varios parámetros: de la composición de cada una de las fases del material cerámico; de la microestructura del material cerámico; de la temperatura del proceso de solicitación; de la composición del agente externo y de la presión y atmósfera en las cuales el material cerámico está inmerso.

\section{PROCESO EXPERIMENTAL}

Para el estudio de la corrosión en los materiales refractarios del horno durante la sinterización de aceros al Mn, la empresa Cremer Thermoprozessanlagen GMBH, suministró ladrillos de uso frecuente en los hornos industriales de sinterización.

La primera parte del trabajo consistió en la caracterización de la composición de las muestras de refractario (15) mediante técnicas de microscopía electrónica de barrido (MEB, Phillips XL-30) y de difracción de rayos-X, utilizando un difractómetro automático Philips $\mathrm{X}$ Pert para determinar las especies presentes. La fuente utilizada fue $\mathrm{Cu}(\lambda=1.5418 \AA \AA)$ y el intervalo de barrido en $2 \theta$ fue de $20-110^{\circ}$, con un paso de $0.02^{\circ}$. La composición y homogeneidad de las muestras iniciales se estimó de forma semi-cuantitativa, mediante espectroscopia de energía dispersiva (EDS) sobre muestras molidas (Fig.1). Para reconocer mejor los mecanismos y el proceso de degradación fue necesario medir la densidad aparente de los mismos mediante pesada, y con ello tener definida la porosidad de estos.

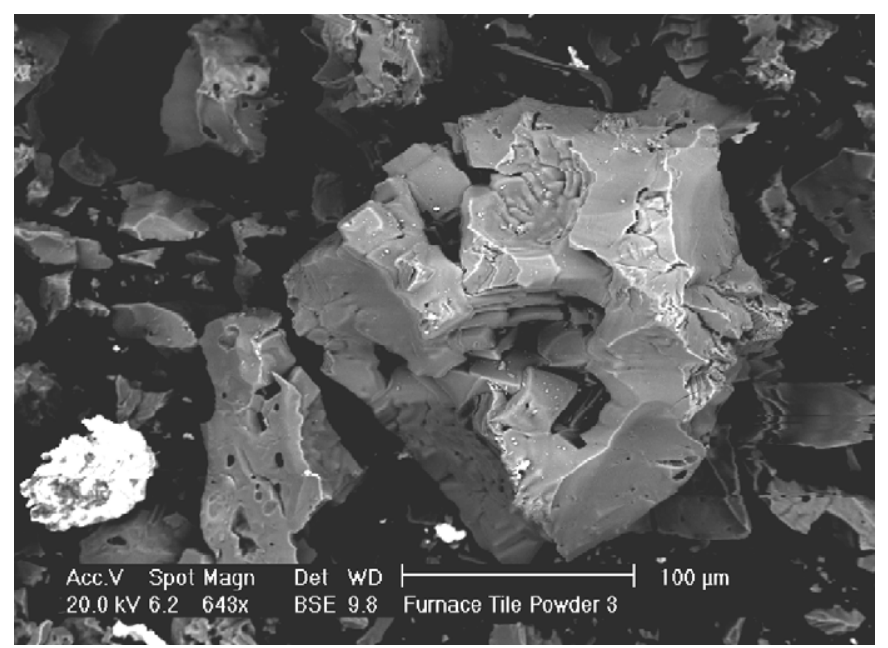

Fig. 1- Micrografía de la muestra molida. 
Aunque en la práctica la cantidad de Mn presente en este tipo de aceros de baja aleación, nunca supera el $2 \%$, el experimento se realizó utilizando una mezcla del $50 \%$ de Fe y $50 \%$ de Mn con objeto de acelerar el proceso y así visualizar el mecanismo de corrosión. Para la evaluación de la degradación de los refractarios se preparó una mezcla 50:50 de polvo Fe atomizado en agua (ASC100.29, calidad de Höganäs AB) y de Mn electrolítico (de entre $-45 \mathrm{y}+125 \mu \mathrm{m}$ ) -agente externo- que se dispuso en una barquilla de alúmina densa con $99,97 \%$ de pureza, tapada por la muestra del refractario problema (Fig.2). El conjunto se mantuvo a $1120^{\circ} \mathrm{C}$ durante $3 \mathrm{~h}$ en atmósfera de $\mathrm{N}_{2}-10 \mathrm{H}_{2^{\prime}}$ empleada habitualmente en la sinterización de este tipo de aceros, con velocidades de calentamiento y de enfriamiento de $5^{\circ} \mathrm{C} / \mathrm{min}$.
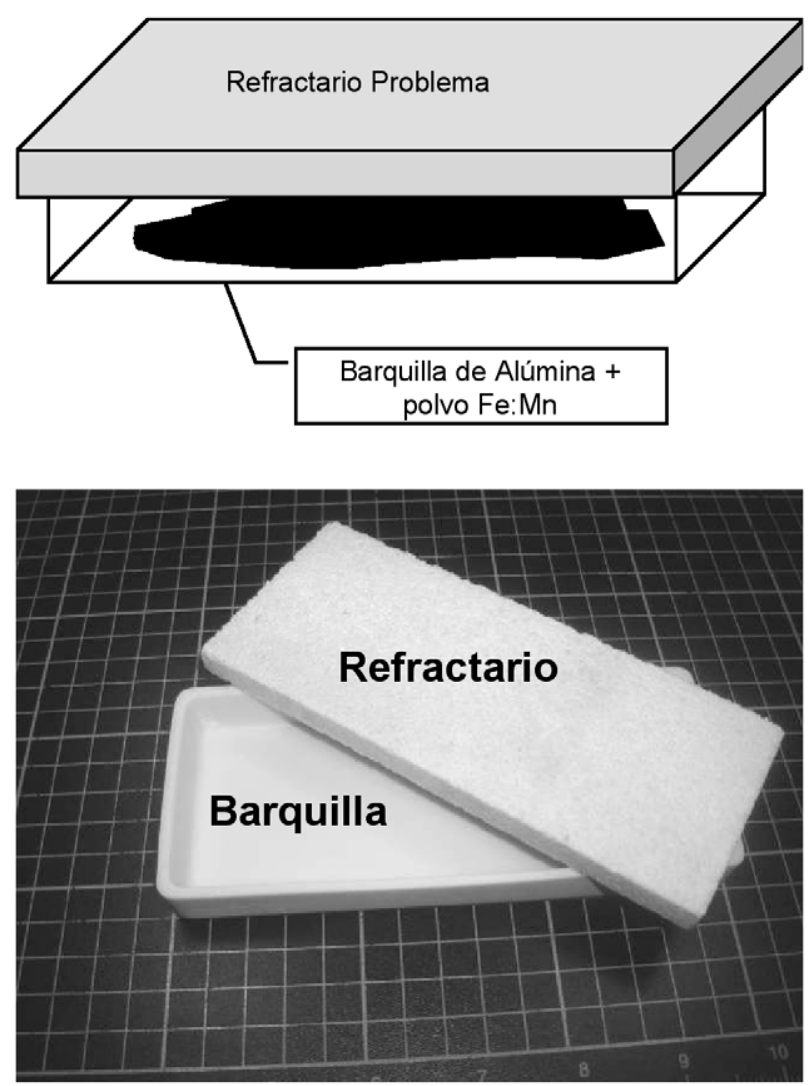

Fig. 2- Esquema de la disposición de las muestras de refractario y del agente externo, para la exposición de los refractarios a la atmósfera de sinterización.

Es necesario conseguir que la disposición del agente externo sea uniforme, por ello la mezcla de Fe y Mn se realizó en Turbula durante $30 \mathrm{~min}$, y se vertió siempre una cantidad de $50 \mathrm{~g}$ sobre la barquilla de alúmina. Con esta configuración se asegura la interacción entre los vapores de Mn y el ladrillo refractario.

Esta mezcla fue renovada cada tres horas de permanencia a la máxima temperatura. Así, se aseguraba la presencia de polvo de manganeso elemental, y que por tanto, se mantuviera por sublimación, en la atmósfera expuesta a la muestra de refractario durante el experimento. De esta forma, se pudo monitorizar el proceso de degradación con el tiempo de sinterización.

El grado de corrosión del ladrillo cerámico se estudió mediante la observación visual; cambios de peso tanto en la muestra de refractario, como en el polvo introducido en una balanza de precisión $( \pm 0.0001 \mathrm{~g})$ a temperatura ambiente; técnicas de microscopía electrónica de barrido tras preparación metalográfica estándar (desbastado con $\mathrm{SiC}$, pulido con diamante y ataque con una disolución acuosa $10 \%$ de HF) y de difracción de rayos-X. Gracias a la fluorescencia de Rayos-X (FRX), se pudo determinar de una manera rápida y con una elevada sensibilidad la composición de la superficie de las muestras expuestas al agente externo, al utilizar la emisión secundaria o fluorescente de radiación de rayos- $X$ generada al excitar una muestra con una fuente de radiación X. El espectrómetro de FRX utilizado fue de la firma PHILIPS modelo X'Unique II.

\section{RESULTADOS Y ANÁLISIS}

\subsection{Caracterización del material refractario inicial}

La muestra suministrada, según los resultados de la difracción de RX, está constituida por corindón de manera exclusiva (Fig.3). Los análisis semi-cuantitivos por EDS (Tabla I) confirman la composición elemental de las muestras

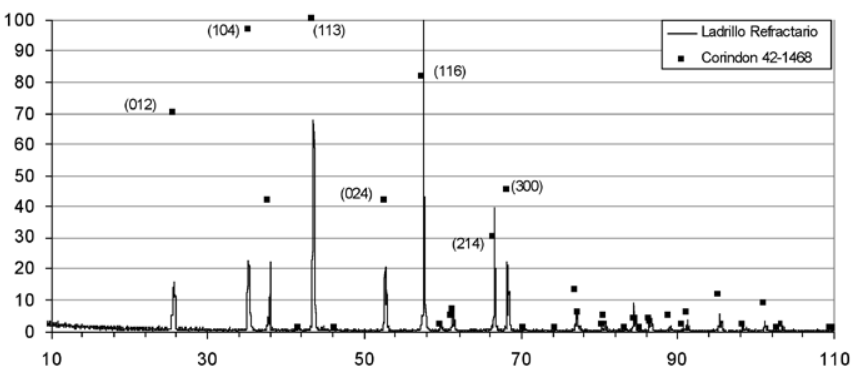

Fig. 3- Espectro de difracción de la muestra de Refractario de alta alúmina. Ficha JCPDS 42-1468.

TABLA I. RESULTADOS DEL ANÁLISIS ELEMENTAL SEMI-CUANTITATIVO MEDIANTE EDS. PARÁMETROS DE CORRECCIÓN ZAF DE NÚMERO ATÓMICO, ABSORCIÓN Y FLUORESCENCIA RESPECTIVAMENTE. RAZÓN K ENTRE LA INTENSIDAD MEDIDA EN LA MUESTRA Y AQUÉLLA DEL ELEMENTO PURO.

\begin{tabular}{|c|c|c|c|c|c|c|}
\hline Elemento & $\begin{array}{c}\% \\
\text { Peso }\end{array}$ & $\begin{array}{c}\% \\
\text { Átomos }\end{array}$ & K-Razón & $\mathrm{Z}$ & $\mathrm{A}$ & $\mathrm{F}$ \\
\hline $\mathrm{Al}$ & 60.15 & 47.37 & 0.4613 & 0.9712 & 0.7896 & 1.0001 \\
\hline $\mathrm{O}$ & 39.33 & 52.24 & 0.1491 & 1.0429 & 0.3630 & 1.0009 \\
\hline $\mathrm{Si}$ & 0.52 & 0.39 & 0.0022 & 0.9995 & 0.4147 & 1.0000 \\
\hline
\end{tabular}

Para el estudio de degradación propuesto, este refractario de corindón es idóneo por su simplicidad química y su pureza. Así, la determinación de las nuevas fases que se formen durante la sinterización será más precisa.

En la Fig. 4 se muestran dos micrografías obtenidas mediante MEB (microscopía electrónica de barrido) de este refractario. Se observan dos características distintivas: una que es un material conformado mediante sinterización de esferas huecas con una distribución de tamaños ancha, y dos, que además existe una porosidad entre estas esferas elevada. Estos dos factores van a ser cruciales en el estudio de la corrosión ya que la superficie específica de dicho material es elevada con lo que la interacción entre el vapor de Mn y el refractario estará muy favorecida. 

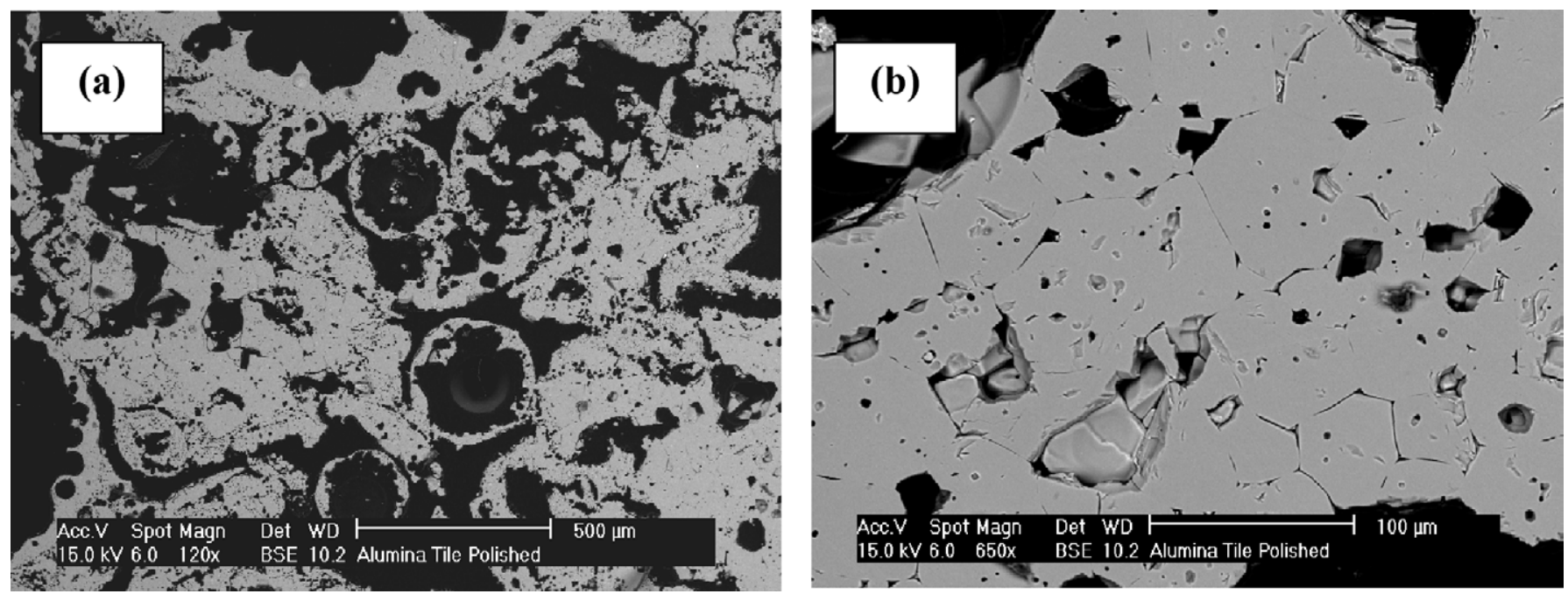

Fig. 4- Micrografías obtenidas mediante MEB de la muestra inicial pulida y atacada 1 segundo con una disolución 10\% HF: a) aspecto general de la muestra, b) detalle de la microestructura de las paredes de las esferas de alumina.

Los valores de densidad aparente corroboran la microestructura altamente porosa observada previamente. Se midieron valores de densidad aparente de $1.42 \pm 0.05 \mathrm{~g} / \mathrm{cm}^{3}$, y sabiendo que la densidad teórica de la corindón es de $3.9 \mathrm{~g} /$ $\mathrm{cm}^{3}$, la porosidad es por tanto del $63 \%$.

\subsection{Degradación del refractario}

Tal y como se ha descrito, el agente externo se renueva cada tres horas para asegurar la presencia $\mathrm{Mn}_{(\mathrm{g})}$ durante el ensayo. Por este motivo, la monitorización del cambio en el peso del ladrillo refractario se ha realizado en intervalos de tres horas. En la Fig. 5 se observa la ganancia de peso de la muestra de ladrillo refractario con el tiempo de exposición al agente externo, manteniendo una tendencia lineal-creciente durante las primeras 18 horas de exposición. Aún siendo escasa la ganancia de peso, la superficie de las muestras refractarias aparece claramente alterada según aumenta el tiempo de sinterización total (Fig. 6). Por otro lado, el incremento de peso sufrido por el agente externo se midió cada tres horas, coincidiendo con la reposición de nuevo agente externo. Este cambio fue de $0.82 \pm 0.34 \mathrm{~g}$, la diferencia es relativamente pequeña y debida fundamentalmente a la oxidación del polvo

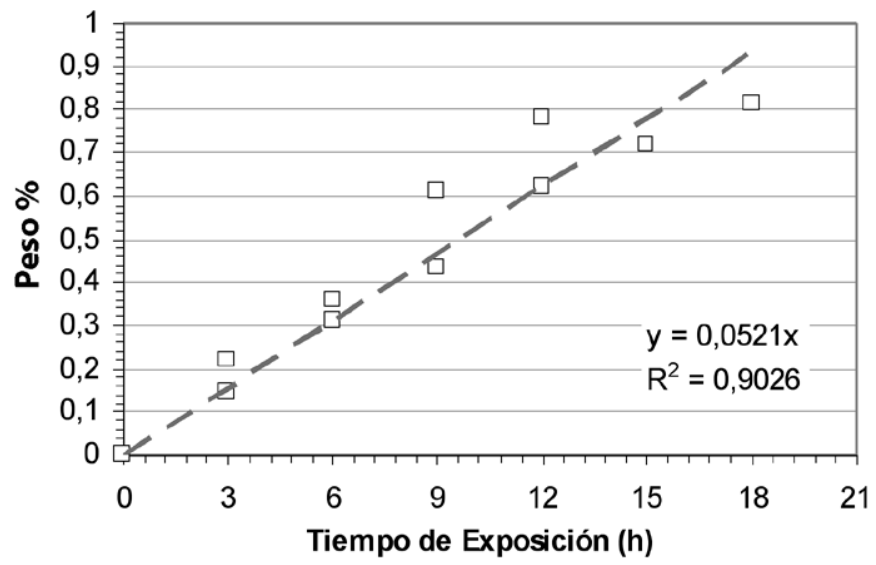

Fig. 5- Evolución del aumento de peso en la muestra con el tiempo de exposición al agente externo. durante el proceso de enfriamiento realizado en $\mathrm{N}_{2}$. Bajo estas atmósferas, no se consiguen los agentes reductores necesarios que eviten la oxidación del polvo de hierro y manganeso restante. Sin embargo, no afecta a los procesos de degradación con la muestra refractaria.
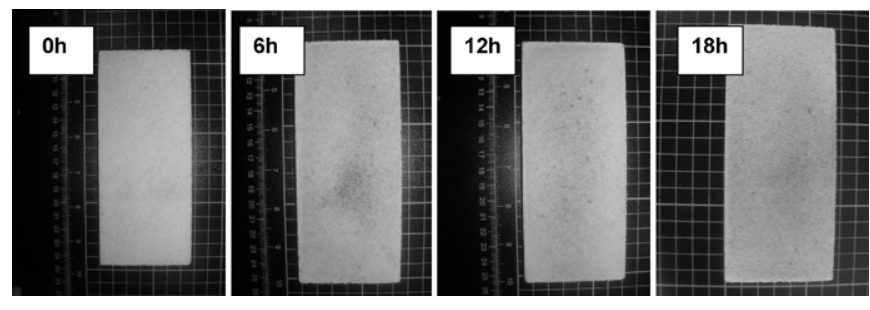

Fig. 6- Evolución del cambio en el aspecto del ladrillo refractario con el tiempo de exposición sinterización.

El cambio en el color de la superficie de las muestras como resultado de la interacción entre el Mn y el corindón es indicativo de la formación de un nuevo compuesto. Para facilitar su determinación, se consideraron los diagramas de fases del sistema binario $\mathrm{MnO}-\mathrm{Al}_{2} \mathrm{O}_{3}(16,17)$ y ternario $\mathrm{MnO}$ $\mathrm{Al}_{2} \mathrm{O}_{3}-\mathrm{SiO}_{2}(18,19)$ mostrado en la Fig. 7.

Teniendo en cuenta el diagrama de equilibrio $\mathrm{MnO}-\mathrm{Al}_{2} \mathrm{O}_{3}$ y mediante los resultados que se han obtenido mediante difracción de RX (Fig. 8) sobre las muestras expuestas a los vapores de $\mathrm{Mn}$, se constata la formación de la espinela $\mathrm{MnO}$. $\mathrm{Al}_{2} \mathrm{O}_{3}$, galaxita, de composición $60 \%$ en peso $\mathrm{Al}_{2} \mathrm{O}_{3}$ y $40 \%$ en peso $\mathrm{MnO}$. Si bien la formación de Galaxita disminuye la temperatura de trabajo del refractario de corindón, la temperatura del punto invariante galaxita-corindón es 1760 $\pm 10^{\circ} \mathrm{C}$, esta es muy superior a la temperatura del experimento $1120^{\circ} \mathrm{C}$. Por otro lado aún teniendo en cuenta las pequeñas impurezas de silicio del refractario de corindón (20) (Tabla I), la temperatura del experimento es muy próxima a la del punto invariante $\mathrm{Al}_{2} \mathrm{O}_{3}-\mathrm{MnO} \cdot \mathrm{Al}_{2} \mathrm{O}_{3}-\mathrm{Mn}_{3} \mathrm{Al}_{2} \mathrm{Si}_{3} \mathrm{O}_{12} \sim 1100^{\circ} \mathrm{C}$ por lo que no es previsible la formación de cantidades significativas de fases líquidas como consecuencia del proceso de corrosión. En la Fig. 7 se han indicado la composición del refractario (R) y la variación de la composición de este refractario como consecuencia del proceso de corrosión mediante una línea 
de puntos. Sobre esta línea se puede observar que las fases esperadas en el material corroído son: corindón, galaxita y una pequeña cantidad de vidrio (fase líquida a la temperatura del ensayo de corrosión).

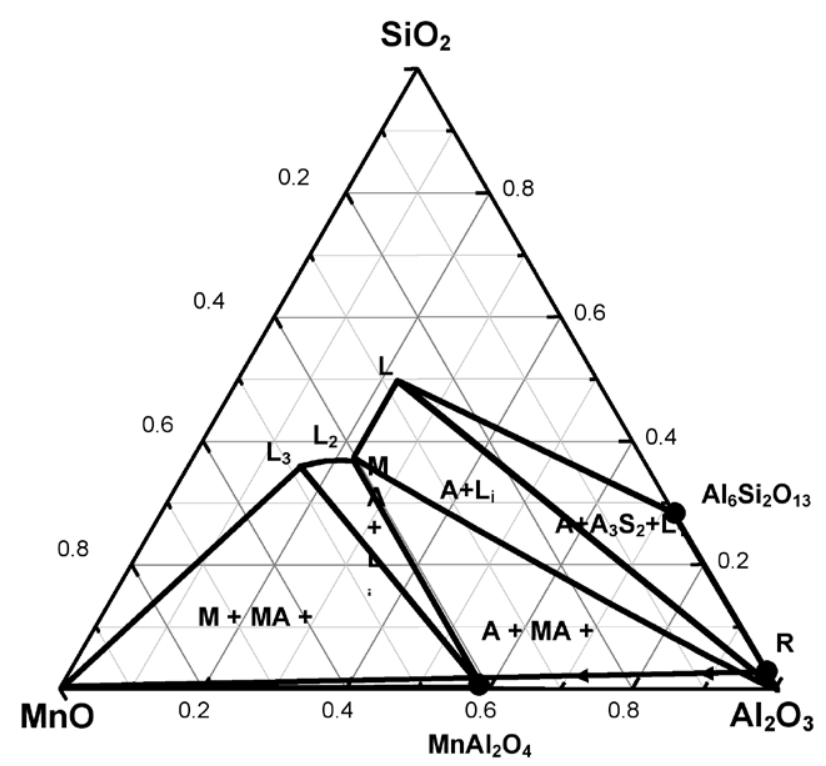

Fig. 7- Esquema del proceso de corrosión partiendo de la composición inicial del refractario estudiado $(\mathrm{R})$ hasta la formación de la espinela de $\mathrm{MnAl}_{2} \mathrm{O}_{4}$.

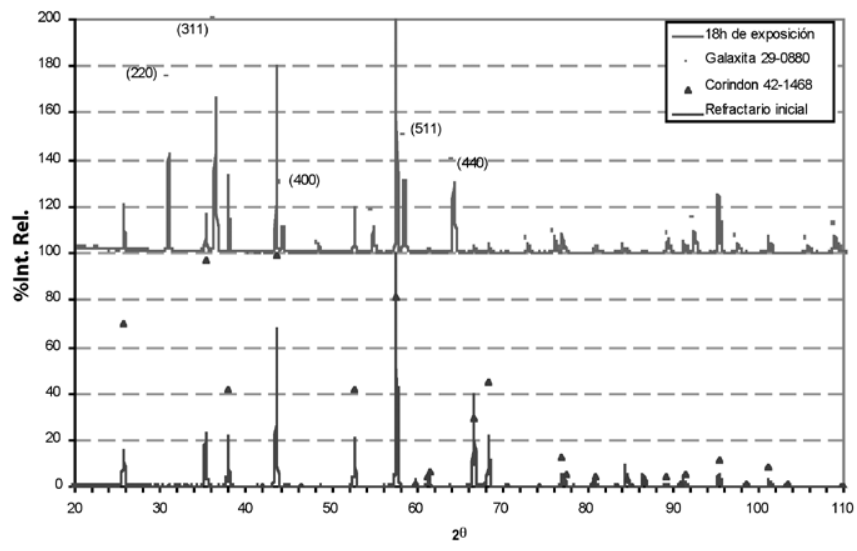

Fig. 8- Arriba Difractograma de la superficie expuesta a $18 \mathrm{~h}$ de sinterización. Abajo Difractograma de la muestra de corindón inicial. Los planos correspondientes a los picos más intensos de la espinela están especificados.

Una vez confirmada la formación de la espinela $\mathrm{MnO}$. $\mathrm{Al}_{2} \mathrm{O}_{3}$ se han de tener en cuenta las diferencias entre las densidades de ambos compuestos (4,088 y $4,16 \mathrm{~g} \cdot \mathrm{cm}^{-3}$ para el corindón y la espinela) los cambios de densidades asociados a la formación de dicha espinela pueden promover la formación de microgrietas superficiales, produciendo un deterioro de la superficie del refractario, que hace aumentar su susceptibilidad a próximos agentes corrosivos.

Por otro lado el corindón y la espinela de manganeso y aluminio (galaxita) tienen diferentes coeficientes de expansión térmica lo que puede originar la formación de grietas en el material durante los procesos de calentamiento y enfriamiento del horno.
No obstante, para determinar la cantidad de Mn que ha difundido en la superficie del refractario de alúmina se realizaron estudios de fluorescencia de rayos $X$. Dichos análisis barrieron una superficie de $20 \times 20 \mathrm{~mm}$ y alcanzaron una profundidad de $250 \mu \mathrm{m}$. Los resultados de dicho estudio se muestran en la Fig. 9. Observando la tendencia que sigue la cantidad de Mn registrada observamos que de nuevo a medida que el tiempo de exposición al agente externo aumenta, también lo hace la cantidad de Mn presente en la superficie del refractario.

Observando más detenidamente los resultados expuestos en la Fig. 9 se puede determinar la cantidad de galaxita formada en el espesor barrido por el análisis de fluorescencia de rayos- $X$. Teniendo en cuenta que la composición en peso de la galaxita es $40 \% \mathrm{MnO}: 60 \% \mathrm{Al}_{2} \mathrm{O}_{3^{\prime}}$ y sabiendo los porcentajes de cada elemento obtenidos tras el análisis de FRX, se puede deducir que tras 18 horas de exposición el agente externo ha sido capaz de penetrar en la superficie del refractario lo suficiente como para que el análisis de FRX detecte sólo Galaxita.

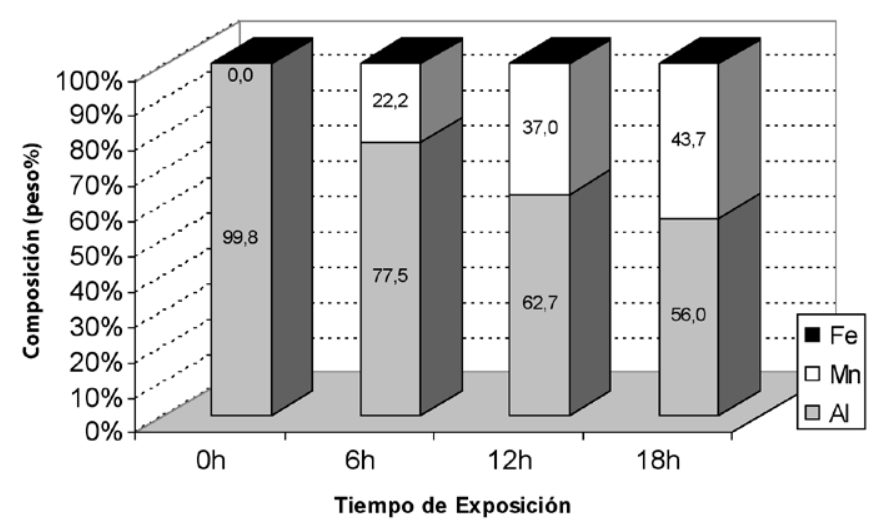

Fig. 9- Evolución de la difusión del Mn sobre la superficie del refractario con el tiempo de exposición.

\section{CONCLUSIONES}

El vapor de Mn que se produce durante la sinterización de aceros que contienen Mn elemental interacciona con los refractarios del horno. En el caso estudiado, el Mn reacciona con la superficie del refractario de corindón formando la espinela Galaxita $\left(\mathrm{MnO} \cdot \mathrm{Al}_{2} \mathrm{O}_{3}\right)$. Dado el estado en el que se encuentra el agente externo, el mecanismo de corrosión es por difusión del vapor de Mn a través de los bordes de grano del material refractario. Además, se ha observado que el incremento en peso del ladrillo refractario, debido a la formación de dicha espinela, sigue una evolución lineal con respecto al tiempo de exposición al agente externo.

Finalmente, se han de tener en cuenta los cambios de volumen asociados a la formación de la Galaxita en la superficie de los refractarios de corindón, y las diferencias entre los coeficientes de dilatación de la galaxita y el corindón.

\section{AGRADECIMIENTOS}

Los autores agradecen a la III Cátedra Höganäs en Pulvimetalurgia por el asesoramiento científico durante el desarrollo de este trabajo, a Cremer Thermoprozessanlagen 
GMBH (Alemania) por el suministro de los ladrillos refractarios y finalmente a HidroNitro SA por el suministro de Mn electrolítico.

\section{BIBLIOGRAFÍA}

1. A. H. DE Aza, P. Pena, S. DE Aza, M. Raigón, J. Gómez-Millán, C. Domínguez, Estudio post-mortem de un hormigón refractario colocado en la línea de escorias de una cuchara de acería, Bol. Soc. Esp. Ceram.V., 42, 3, 375-378 (2003).

2. M. Campos, D. Sánchez, J.M. Torralba, Sintering behaviour improvement of a low Cr-Mo prealloyed powder steel through Mn additions and other liquid phase promoters, J. Mater. Proc. Tech. 143-144 C, 464-469, (2003).

3. M.Campos; S. Kremel, T. Marcu Puscas, Y. Yu, D. Sánchez, J.M. Torralba, Effect of heat treatments on Cr-Mo steels based on Astaloy CrM powder grade with Manganese additions, Mater. Sci. Forum 416418, 82-88, (2003)

4. J. Sicre-Artalejo, M. Campos, T. Marcu, JM Torralba, Modification of Low Alloyed Steels by Manganese Additions, Mater. Sci. Forum., 534-536, 697-700, (2007).

5. M. Campos, L. Blanco, J. Sicre-Artalejo, J.M. Torralba, Aceros de baja aleación y alto rendimiento, Revista de Metalurgia Vol. 44 (1), 5-12 (2008).

6. G. Zapf, G. Hoffman, K. Dalal, Effect of Additional Alloying Elements on the Properties of Sintered Mn Steels, Powder Met., 18, 35, 214-236, (1975).

7. A. Salak, Sintered Manganese Steels I: Effect of Structure of Initial Iron Powders Upon Mechanical Properties, Powder Metall. Int., 12, 1, 28-31, (1980).

8. A. Salak, Sintered Manganese Steels. II: Manganese Evaporation During Sintering, Powder Metall. Int.,12, 2, 72-75, (1980).
9. A. Salak, Manganese Vapor-Protection of Premixed Manganese Steels Against Oxidation During Sintering, Powder Metall. Int., 18, 4 266-270, (1986).

10. G.S.Upadhyaya, Manganese in Powder Metallurgy Alloys, The Manganese Centre ed., Paris, (1986)

11. A. Cias, S.C. Mitchell, K. Pilch, H. Cias, M. Sulowsky and A.S Wronsky, Tensile properties of Fe-3Mn-0.6/0.7C steels sintered in semiclosed containers in dry hydrogen, nitrogen and mixtures thereof, Powder Metall.3, 46, 2, 165-170, (2003).

12. E. Otero, Corrosión y Degradación de Materiales, Capítulo 24. Ed Síntesis SA, Madrid, (1997).

13. A.H. de Aza, Corrosión de materiales refractarios por escorias y vidrios fundidos, Bol. Soc. Esp.Ceram.Vidrio, 35, 2, 87-101, (1996).

14. Tsuchida, T., Hasegawa, T., Kitagawa, T. and Inagaki, M., Aluminium nitride synthesis in air from aluminium and graphite mixtures mechanically activated, J Eur Ceram Soc, 17, 15-16, 1793-1795 (1997).

15. A.H. De Aza, F., J. Valle, P. Ortega, P. Pena, S. De Aza, Analytical Characterization of a Magnesia-Graphite Refractory, J. Am. Ceram. Soc., 89, 5, 1704-1708, (2006).

16. Gunnar Eriksson, Ping Wu and Arthur D. Pelton, Critical evaluation and optimization of the thermodynamic properties and phase diagrams of the MgO-Al2O3 MnO-Al2O3, FeO-Al2O3, Na2O-Al2O3 and K2OAl2O3 systems, CALPHAD 17, 2, 189-205, (1993).

17. In-Ho Jung, Youn-Bae Kang, Sergei A. Decterov, And Arthur D. Pelton, Thermodynamic Evaluation and Optimization of the MnO$\mathrm{Al} 2 \mathrm{O} 3$ and $\mathrm{MnO}-\mathrm{Al} 2 \mathrm{O} 3-\mathrm{SiO} 2$ Systemsand Applications to Inclusion Engineering, Metall. Mater. Trans. B. 262, 35B, 259-268 (2004).

18. United States Patent number: 5,314,549, High Strength and High Toughness Stainless Steel Sheet and Method for Producing thereof, May 1994.

19. Ghasem Roghani, Evgueni Jak, And Peter Hayes, Phase-Equilibrium Studies in the "MnO"-A12O3-SiO2 System. Metall. Mater. Trans. B, 33, 6, 827-838, (2002)

20. Zhou, J. Bahadur, S., Erosion characteristics of alumina ceramics at high temperatures, Erosion, Wear, 181, 178-188, (1995)

Recibido: 26.11.07

Aceptado: 23.06 .08 